\title{
THE REALIZATION NETWORK CORRESPONDENCE WITH WINSOCK CONTROL COMPONENT OF VB
}

\author{
Chunjiang Zhao ${ }^{1}$, Cheng Wang ${ }^{1}$, Jun Yang ${ }^{1,2,{ }^{*}}$, Xiaojun Qiao ${ }^{1}$ \\ I National Engineering Research Center for Information Technology in Agriculture, china, \\ 100097 \\ 2 China Agricultural University, Beijing, China, 100083 \\ * Corresponding author, Address: Shuguang Huayuan Middle Road 11\#, National Engineering \\ Research Center for Information Technology in Agricultur, Beijing, China, 100097, Tel: +86-10- \\ 51503409, Fax: +86-10-51503449, Email: yang1jun2@sohu.com
}

Abstract: This paper mainly expounds that using the WINSOCK controller which is provided by VB6.0 to program the procedures in the coop intelligent control system, it introduces the character of the TCP protocol and the UDP protocol, and according to the demands of the system environment to choose the suitable protocol. This system uses the WINSOCK controller and the UDP protocol for network communications, and also it has the communication function of network data between all the acquisition controllers and PC.

Keywords: Network Communications, Environment control, Winsock control component

\section{INTRODUCTION}

With the emergence of large-scale, intensive rearing methods, information technology has been widely applied to the aquaculture industry. Foreign environmental control technology for the earlier study, it began in the 1970s, using a combination of analog instrumentation to collect site information to instruct, record and control. In the end of 1980s, distributed control system developed. Now the research and development is multi-factor integrated control system of computer data acquisition and control system. On the domestic, coop environmental monitoring system mostly used the traditional on-site control of RS485, CAN and so on ${ }^{[1-2]}$. we sleeted a technology 
program with universal, scalability and upgrading in hardware and software systems of environment intelligent control system, and it adapt to the domestic situation. All of these make the research and development of coop environmental monitoring technology to have good expansion and upgrading foreign coop environmental monitoring system exists the shortcomings of expensive, incompatible with the status of domestic. To change this situation, According to the coop environment and the system's needs, We embed UDP network protocol in our systems, through the network to make a real-time monitoring to coop, greenhouses and other agricultural installations and environment. By the way, we construct the network data transmission system of coop intelligent monitoring system.

\section{WINSOCK CONTROL NETWORK PROGRAMMING}

In the coop intelligent monitoring system, including acquisition module, transmission module, and analysis modules of environment parameters. In this paper, we introduct the network transmission of environment parameters based on the UDP protocol. In our reasearch of network applications using VB, Winsock Control was used to achieve function of system it is visible to users. It provide a convenient way to visit TCP and UDP network services. Microsoft Access, Visual Basic, Visual C + + or Visual FoxPro developers all can use it. Winsock Control prepares the client and the server application procedures. Without understanding the details of TCP or calling Junior Winsock API. By setting the control attributes and calling its methods can easily connect to a remote computer, and can also achieve a two-way exchange of data ${ }^{[3]}$.

\subsection{Method of Winsock control}

Listen: Server to be used for creating socket and it is set to interception pattern, waiting for called by customer.

Connect: Clients send request to the server.

Accept: Server aware of the connection request of Client, Using this method to agree to connect.

SendData: Data will be sent to the other part.

GetData: Receiving the data come from the other side of the information and storing the data.

Close: Close the using link. 


\subsection{Event of Winsock control}

ConnectingRequest: The incident occurred when Server "listen" to the connect request from client, at this time Server should express receiving or not.

DataArrival: The incident occurred if the other sides send data by the method SendData. At this point these data was deal with, For example, saved it to the database or displayed it.

\subsection{Property of Winsock control}

LocalPort: For the sever, Setting up a local port, approach "listen" listen to news from local port.

RemotePort: Customers set up a the remote port to visit, namely the server localport value.

RemoteHost: Clients designated computer to connect, namely IP address. Like "192.168.4.144", we also can use the computer name "admin".

State: The states of Winsock Control, namely the linking of two state machines.

Protocol: Returning or setting up protocol of Winsock Control used (TCP or UDP).

\section{NETWORK COMMUNICATION PROTOCOL}

In Internet, the main protocol is the TCP/IP protocol,there has two types of protocol to achieve information transmission in TCP/IP transport layer, They were the TCP and $\mathrm{UDP}^{[4]}$. Characteristics of TCP and UDP were introduced as following.

\subsection{TCP Basis}

TCP is a connection-oriented protocol, the protocol that need to establish a reliable link between the two points, Which includes a special mechanism to ensure the transfer, When the receiver has received reports from the sender information and automatically send to information confirmed to sender. The sender continue to send other information after receiving confirmation of information, Otherwise the sender will have to wait until the information of 
confirmed received. TCP used for non-real-time data business normally, it can be compared with the telephone system. At the beginning of data transmission, users must establish a connection.

Data Transfer Protocol allows to creat and maintenance connecting with the remote computer, Two computers can be connected with each other for data transmission. If client applications was created, we must know the server computer name or IP address, We must also know the "bugging" of the port, then called connect Method. If the server application procedures was created, we should set up a "listen" ports, and called listen Methods. The client computer will need to connect when the ConnectionRequest of incident occurred to complete the connection, called the incident ConnectionRequest of Accept Method. After establishing a connection, any one computer can be send and receive data, in order to send information, called the SendData of method. When receiving data, DataArrival incident occurred. Called the incident DataArrival GetData method to get data.

\subsection{UDP Basis}

UDP is a news-oriented protocol, it does not need to establish communication link and not provide data transmission mechanism of the guarantee. If the data was lost from the sender to the receiver of the transfer process, the protocol itself does not make any detection or prompt. So UDP is known as unreliable transmission protocol. Between the two computers similar to the transmission of mail transmission, message is sent from a computer to another computer, but the two is no clear link. In addition, each transmission of the largest volume of data depended on the specific network.

UDP is a connectionless protocol, UDP is different operation of TCP. It is not establish a link between computers. In addition, the UDP applications procedure can be both client and server. To transmit data, we must first set up a client computer's LocalPort attributes. Then, the server computer can be set up RemoteHose as customers computer Internet address. RemotePort attribute was set to the same as client computer with LocalPort port, called SendData Method to send information. Client computer have access to the sended information using GetData method of DataArrival incident.

\section{PROTOCOL CHOICE}

In the use of Winsock, the first we must consider the kind of communication protocol. Network for data communications, it needs to mark the mainframe network using address, in order to ensure correct data sent to the mainframe. We have introduced the use of the protocol. Including TCP and UDP protocol, the difference between the two protocols is that their 
connection status, TCP is a connection-oriented protocol, and UDP is a connectionless protocol ${ }^{[3]}$.

In the system design which protocol should be chosen in the end, protocol was choosed by Establishing Application Programm. Following several aspects of the introduction and analysis of our system suitable agreement.

(1) At the time of sending and receiving data, application procedures, whether they need to be client or server confirmation message? If you need to use TCP, the sending and receiving data before establishing a clear link to ensure reliability

(2) Transmission data whether it is particularly large (like images and audio files)? If yes, after connected, TCP protocol can safeguard connection and ensure the integrity of data, but, this link requires more computing resources, so it is more "expensive" in Cost and efficiency.

(3) Whether this data is sent the interval in a conversation or completed? If Application procedures required to notify a computer in a particular task completed, then UDP is more appropriate, it is suitable for a small amount of data to send or immediate data.

Through the analysis we have come to this conclusion, UDP protocol suitable for the two sides what do not need confirmation communication of information, UDP has small resource consumption, with kept its speed more advantage than TCP. Although TCP protocol was implanted various security functions, TCP will take up a lot of consumption cause speed to be severely affected in actual executive process. UDP excluded the reliability of information transmission mechanism and transferred to the upper application to complete security and scheduling of protocol greatly reducing the execution time, the speed of a guarantee, Although UDP protocol lost some packets in application, the receiver will not have much impact on the results. Comparing with the reliability example, in the practical application, We pay more attention to the actual performance, so in order to obtain better performance, we can sacrifice some reliability.

Therefore, after considering the effect of communication UDP, speed and the requirements of environmental facilities, we adopted UDP protocol between controllers in the environmental monitoring system. Any one collector can send data to any other collector, without getting confirmed by other information, Using broadcasting, a collector broadcast their own data to other collector. In Practical, all collectors collect information outside the station also, namely information of station is shared by all controllers as a reference to the factors.

Comprehensive analysis, the system adopted linking-less of UDP, with support of network communication between PC and controller, the network communication structure shown in the following. 


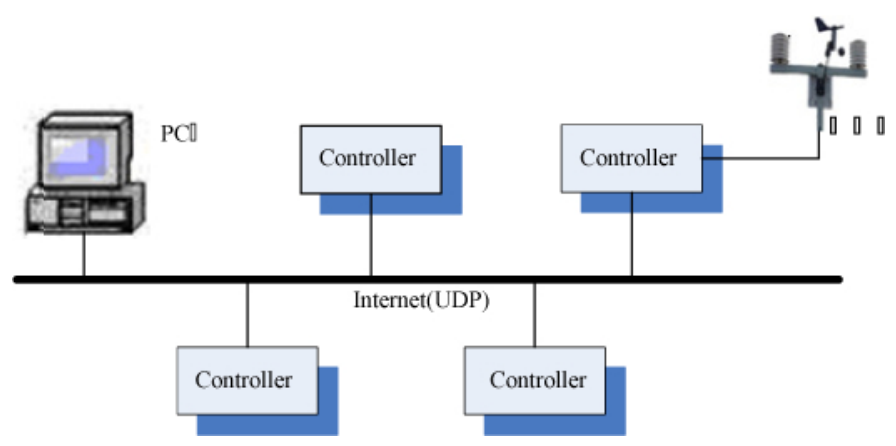

Fig. 1. The structure chart of the network communication

Controller equivalent to a PC in the network in system, System can assign an IP address for each controller. Not only Controller exchanged data with pc, but also realized data sharing between the controllers. The station information as a shared data can publish information in form of broadcasting in network. Other network nodes can be set up monitoring ports in network and monitor different types of data; we can set up port what is corresponding to weather station nodes, in order to receive the data and data sharing.

UDP must know device's IP address and port number in the process of communication. We can read all the information in Initialization procedure. we know to who communication and read out its corresponding IP and Port in the process. In order to transmission of data between multi-machine, we must set up separately LocalPort for each attribute, we set up Remote Host attribute of PC as Controller IP addresses and Remote Port attribute of PC as controller LocalPort attribute value. Then PC called SendData method to realize transmission of data. Controllers use GetData method of DataArrival to obtain information what $\mathrm{pc}$ sent to controller. If controller send data to $\mathrm{PC}$, the process is the same as above, some codes are as follows:

frmMDIMain.WinsockUDP.RemoteHost=typDevice(iDevice). iRemoteHo st /* seting up RemoteHost attribute of PC as Controller IP addresses

frmMDIMain.WinsockUDP.LocalPort $=1234 / *$ Setting up local port

frmMDIMain.WinsockUDP.RemotePort $=$ typDevice(iDevice).iRemotePort/*setting up RemotePort attribute of PC as controller LocalPort

frmMDIMain.WinsockUDP.SendData string /*calling SendData method

Private Sub WinsockUDP_DataArrival(ByVal bytesTotal As Long)/* Calling the incident DataArrival GetData method to access data

Dim rec As String

WinsockUDP.GetData rec, vbString

ReceiveBufferUDP $=$ rec

ReceiveOK $=$ True

End sub 


\section{CONCLUSIONS}

The system has realized the sheds environmental parameters monitoring network in Environmental parameters of the transmission using UDP data transmission network. In the coop, the system adopted a network communication Technology between controllers, as well as between the controller and PC, namely UDP protocol. UDP protocol can greatly enhance communication efficiency and lift velocity. UDP protocol can greatly reduce the polling time and reduce the execution time when pc collected

Data form collector. The purpose of systems development makes environmental management of chicken house toward more intelligent and more network in the direction.

\section{ACKNOWLEDGEMENTS}

This research is supported by the National High Technology Research and Development Program of China (863 Program, Grant 2006AA10A311 and Grant 2006AA10Z253) and Beijing Science \& Technology Program (Z0006321001391).

\section{REFERENCES}

ChenWei, Shixi Yang. Visual Basic Programming from basic to practice. Beijing: electronic industrial press, 2005:210-271.

Deze Zhou, Naner Yuan, Yingying. The Design and Application of Computer intelligent Control System. Beijing: Tsinghua University press, 2002:57-60.

Changhua Lu, Wuzi, Lifang Wang etc. Establishment and application of computerized production management system for large scale poultry farm. Journal of Agricultural Engineering, 2003, 19 (6):256-259.

Niuli. Visual Basic6.0 programming. Beijing: electronic industrial press, 2005.

Yadong Luo. Implementation of network real-time communication with Winsock. Chengdu Information Technology Institute, 2004, 19(2):198-201.

Yinong Hu, Shaochun Dou, Lifang Wang etc. Hens of scale farms with Automatic Monitoring System. Jiangsu Agriculture Journal., 2002, 18(3):176-180.

Zhou Juan, Xianghua Chen. With Winsock UDP-based establish applications procedure In VB. Electronic machinery College Journal of Chengdu, 2005, 31(2):17-20. 